\title{
Pleuroparenchymal fibroelastosis: a spectrum of histopathological and imaging phenotypes
}

\author{
Taryn L. Reddy*, Masaki Tominaga", David M. Hansell*, Jan von der Thusen\#, \\ Doris Rassı ${ }^{\ddagger}$, Helen Parfrey ${ }^{+}$, Suzy Guy ${ }^{\S}$, Orion Twentyman ${ }^{f}$, Alexandra Rice ${ }^{\#}$, \\ Toby M. Maher**, Elisabetta A. Renzoni**, Athol U. Wells** and Andrew G. Nicholson ${ }^{\#, * *}$
}

ABSTRACT: Pleuroparenchymal fibroelastosis (PPFE) is a rare condition characterised by predominantly upper lobe pleural and subjacent parenchymal fibrosis, the latter being intraalveolar with accompanying elastosis of the alveolar walls. The aim of this study was to review cases fulfilling published imaging and histological criteria, and identify any common clinical features that may suggest an underlying aetiology for a condition that has previously been regarded as idiopathic.

Of 12 patients (seven females, median age $57 \mathrm{yrs}$ ), the presenting symptoms were shortness of breath (11 out of 12 patients) and dry cough (six out of 12 patients). Seven patients reported recurrent infections during the course of their disease. Five demonstrated nonspecific autoantibody positivity. Two patients had a family history of interstitial lung disease (ILD).

High-resolution computed tomography features of lung disease remote from the pleuroparenchymal changes were present in six out of 12 patients (coexistent fibrosis, $n=5$; bronchiectasis, $n=1$ ). Of seven patients with tissue sampled from the lower lobes, four patients showed less intense PPFE changes (one with additional features of hypersensitivity pneumonitis) and three showed usual interstitial pneumonia.

PPFE is a distinct clinicopathological entity, with clinical data suggesting a link to recurrent pulmonary infection. Genetic and autoimmune mechanisms may also contribute to the development of these changes. PPFE may also present with more diffuse involvement than previously reported, and coexist with different patterns of ILD.

KEYWORDS: Infection, interstitial lung disease, lung, pleuropulmonary fibroelastosis

$\mathbf{P}$ leuroparenchymal fibroelastosis (PPFE) is a rare disorder, first described in the Japanese literature by AMITANI et al. [1] as idiopathic pulmonary upper lobe fibrosis. PPFE comprises dense established intra-alveolar fibrosis, with the alveolar walls in these areas showing prominent elastosis, and dense fibrous thickening of the visceral pleura; these changes have a striking upper-zone predominance $[2,3]$. Little is known regarding its aetiology and most cases are considered idiopathic, although a few cases are familial $[1,2,4-6]$ and others have been reported in association with previous bone marrow transplantation [7]. Furthermore, most reports in the English language post-date the 2002 American Thoracic Society (ATS)/European Respiratory Society (ERS) consensus classification of idiopathic interstitial pneumonias [8], so there is no agreed definition regarding diagnostic criteria for PPFE, in particular whether it should be regarded as a truly idiopathic entity.

The purpose of this study was therefore to evaluate pathological and radiological findings of patients fulfilling published criteria for PPFE, and to review clinical data with regard to possible aetiology.

\section{METHODS}

The diagnostic pathology archives of the Royal Brompton and Harefield NHS Foundation Trust (London, UK) were searched for cases in which the terms "intra-alveolar fibrosis", "pleuroparenchymal" and "fibroelastosis" were present. 33 cases were identified and microscope slides from 30 cases were reviewed by two pathologists (A.G. Nicholson and J. von der Thusen) using published histological criteria for the diagnosis of
AFFILIATIONS

*Dept of Radiology, NHLI Division, Imperial College,

${ }^{\#}$ Dept of Histopathology, Royal Brompton and Harefield NHS Foundation Trust and NHLI Division, Imperial College,

**Dept of Medicine, NHLI Division, Imperial College, London,

'Dept of Histopathology, University of Cambridge and Papworth Hospital NHS Foundation Trust,

${ }^{+}$Dept of Medicine, University of Cambridge and Papworth Hospital NHS Foundation Trust, Cambridge,

${ }^{\S}$ Dept of Respiratory Medicine, Mater Hospital, Belfast, and

fDept of Respiratory Medicine, Norfolk and Norwich University Hospitals NHS Foundation Trust, Norwich, UK.

CORRESPONDENCE

A.G. Nicholson

Dept of Histopathology

Royal Brompton Hospital

Sydney St

London SW3 6NP

UK

E-mail: a.nicholson@rbht.nhs.uk

Received:

Sept 222011

Accepted after revision:

Nov 302011

First published online:

March 222012 
PPFE [2, 3]. These were subsequently characterised by consensus as "definite PPFE", "consistent with PPFE" or "inconsistent with PPFE". "Definite" was assigned when there was upper zone pleural fibrosis with subjacent intra-alveolar fibrosis accompanied by alveolar septal elastosis. "Consistent with" was assigned when intra-alveolar fibrosis was present but it was not 1) associated with significant pleural fibrosis, 2) not predominantly beneath the pleura or 3) not in an upper lobe biopsy. "Inconsistent with" was assigned for cases that lacked the requisite features described above. Of the 30 patients with histological material, 21 patients had high-resolution computed tomography (HRCT) images available for review, which was undertaken by two radiologists (D.M. Hansell and T.L. Reddy). These were independently characterised in a similar fashion to the microscopy samples, based on reported HRCT features from previous case studies that had been histologically confirmed [2,3]. "Definite" was assigned for cases that demonstrated pleural thickening with associated subpleural fibrosis concentrated in the upper lobes, with involvement of lower lobes being less marked or absent. "Consistent with" was assigned for cases where upper lobe pleural thickening with associated subpleural fibrosis was present but 1) distribution of these changes was not concentrated in the upper lobes or 2) there were features of coexistent disease elsewhere. Diseases in other lobes were documented in accordance with the 2002 ATS/ERS Consensus Classification of the Idiopathic Interstitial Pneumonias and 2011 ATS/ERS/ Japanese Respiratory Society/Asociación Latinoamericana de Tórax Evidence Based Guidelines for Diagnosis and Management of Idiopathic Pulmonary Fibrosis [8, 9]. "Inconsistent with" was assigned for cases that lacked the requisite features described above. Cases were only included when they were considered definite or consistent with PPFE by both histopathology and radiology. If either pathologists or radiologists categorised appearances as inconsistent with a diagnosis of PPFE, the case was excluded.

There were 17 out of 30 and 14 out of 21 cases that were definite or consistent with PPFE for histopathology and imaging, respectively. Four cases were excluded from the 17 accepted on histological criteria due to an absence of computed tomography for review, while an additional case was excluded because it did not fulfil imaging criteria (inconsistent with) for PPFE. Of the 14 cases with available HRCTs, two cases were excluded as they did not fulfil the histological criteria, these cases revealing allergic bronchopulmonary aspergillosis $(n=1)$ and a purely bronchocentric intra-alveolar fibrosis $(n=1)$. Accordingly, 12 cases made up the final study group and clinical data for these were retrieved from either the patients' notes or the Electronic Patient Record database.

\section{RESULTS}

\section{Clinical data}

Clinical details and investigations are summarised in tables 1 and 2. Of the 12 patients who fulfilled the inclusion criteria, seven were female and five were male (age range 24-85 yrs, median $57 \mathrm{yrs}$ ). Presenting symptoms included shortness of breath $(n=11)$ and dry cough $(n=6)$; the duration of symptoms prior to diagnosis was 6 months to 6 yrs (mean 2 yrs 8 months). Seven patients reported recurrent lower respiratory tract infections, all having at least three acute episodes prior

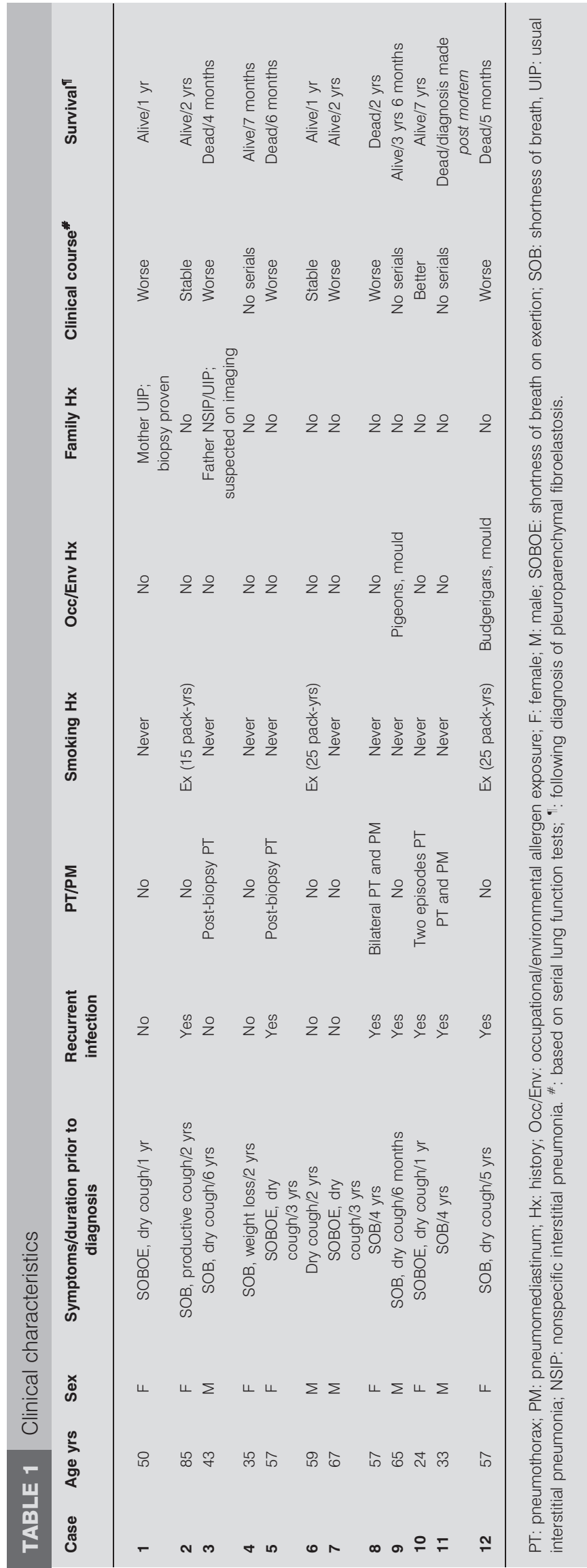




\section{TABLE 2 Laboratory data}

\begin{tabular}{|c|c|c|c|c|c|}
\hline 2 & RF positive & Not performed & Not performed & Restrictive & Not performed \\
\hline 3 & Antiribosomal Ab positive & No & No & Restrictive & 73 \\
\hline 4 & No & & & Restrictive & 31.2 \\
\hline 6 & No & & & Restrictive & 55 \\
\hline 7 & No & No & No & Restrictive & 57.1 \\
\hline 8 & $\begin{array}{l}\text { ANF-IF speckled nuclei, equivocal dsDNA Ab, } \\
\text { cardiolipin IgM Ab positive }\end{array}$ & No & IgG positive & Restrictive & 35 \\
\hline 9 & No & No & No & Normal & 107.7 \\
\hline
\end{tabular}

pptn: precipitin; $T L, C O$ : transfer factor of the lung for carbon monoxide; RF: rheumatoid factor; Ab: antibody; ANCA: anti-neutrophil cytoplasmic antibody; CCP: cyclic citrullinated peptide; ANF-IF: anti-nuclear factor immunofluorescence; dsDNA: double-stranded DNA; Ig: immunoglobulin.

to diagnosis. Three patients developed spontaneous pneumothorax or pneumomediastinum during the course of their disease. Two patients reported exposure to environmental allergens in the form of mould and birds; one of these patients tested negative for mould and avian precipitins, the other patient did not undergo testing. First-degree relatives of two patients were known to have interstitial lung disease (ILD) (biopsy-proven usual interstitial pneumonia (UIP), $n=1$; radiologically suspected nonspecific interstitial pneumonia (NSIP)/ UIP, $n=1$ ). With the exception of one patient (immunosuppressed following a renal transplant for anti-neutrophil cytoplasmic antibody-positive glomerulonephritis), no significant drug history was evident in the study group. Specifically, there was no history of radiotherapy or chemotherapy. None of the patients had undergone bone marrow transplantation. Four of the seven patients who reported recurrent infections also demonstrated autoantibodies in their serum. One of the patients who tested positive for Aspergillus immunoglobulin G antibodies (table 2) had a small focus of bronchocentric granulomatosis superimposed upon the PPFE on histological examination of the lung biopsy. Four of the five patients with autoantibodies had imaging features of distant interstitial fibrosis, either present at initial review (cases 2, 3 and 8) or developing subsequently (case 5). One patient had been diagnosed on a previous hospital admission with Dressler's syndrome (case 7). Of nine patients who underwent bronchoalveolar lavage, one patient demonstrated high levels of eosinophils (case 3). Tuberculosis cultures were negative for all patients.

\section{Imaging data}

Radiological and histopathological features of patients with PPFE are summarised in tables 3 and 4.

Computed tomography findings in all 12 patients revealed bilateral irregular pleuroparenchymal thickening, which was most marked in the upper and middle zones, with an associated subpleural reticular pattern consistent with established fibrosis (fig. 1). Most patients demonstrated subpleural fibrosis of moderate severity (eight out of 12 patients).

Five out of 12 patients demonstrated interstitial fibrosis in regions remote from the pleuroparenchymal changes (excluding case 3, which was considered diffuse (all-zone) PPFE on imaging), with the HRCT pattern of fibrosis being most reminiscent of NSIP/possible UIP (three patients), NSIP (one patient) and unclassifiable interstitial pneumonia (one patient); these patterns were concentrated in the middle and lower zones (figs 2 and 3).

Six patients had small foci of consolidation, all in an upperzone distribution. One patient had co-existent bronchiectasis, and the possibility of underlying allergic bronchopulmonary aspergillosis (ABPA) was raised in this patient, on the basis of the HRCT findings (fig. 4).

Serial computed tomographs were available in six out of 12 patients, and these patients demonstrated stability or minor progression with respect to pleuroparenchymal changes (8-51 months, median 14 months). One patient with interstitial fibrosis at presentation had demonstrated marked progression of fibrosis as judged by a subsequent computed tomography performed 8 months later (case 8). One patient who did not have coexistent interstitial disease on initial review had developed marked lowerlobe fibrosis by the time a subsequent computed tomography was performed 42 months later (case 5).

\section{Pathology data}

Lung biopsies of the upper lobes were undertaken in nine out of 10 patients within 3 months of computed tomography being performed. One patient had biopsies of the lingula and lower lobes, with the lingula showing features consistent with PPFE, and one patient had the diagnosis made at autopsy with all lobes sampled. All patients demonstrated intra-alveolar 


\begin{tabular}{|c|c|}
\hline \multicolumn{2}{|l|}{ Imaging } \\
\hline Pleuroparenchymal thickening in upper zones & $12 / 12$ \\
\hline Pleuroparenchymal thickness mm & $4-15$ \\
\hline \multicolumn{2}{|l|}{ Other upper zone changes } \\
\hline Subjacent fibrosis & $12 / 12$ \\
\hline Mean severity of fibrosis ${ }^{\#}$ & 2 \\
\hline ILD pattern in other zones & $6 / 12$ \\
\hline Diffuse PPFE & $1 / 12$ \\
\hline $\mathrm{NSIP}^{+}$ & $1 / 12$ \\
\hline NSIP/possible UIP & $3 / 12$ \\
\hline Unclassifiable interstitial pneumonia ${ }^{f}$ & $1 / 12$ \\
\hline \multicolumn{2}{|l|}{ Other features } \\
\hline $\mathrm{PAH}^{\# \#}$ & $1 / 12$ \\
\hline Bronchiectasis & $1 / 12$ \\
\hline Consolidation & $6 / 12$ \\
\hline Asbestos-related calcified pleural plaques & $0 / 12$ \\
\hline \multicolumn{2}{|l|}{ Pathology } \\
\hline Pleuroparenchymal fibrosis "ฯ & $12 / 12$ \\
\hline Pleural fibrosis & $11 / 12$ \\
\hline Interstitial elastosis & $12 / 12$ \\
\hline IAF & $12 / 12$ \\
\hline \multicolumn{2}{|l|}{ Other changes } \\
\hline Bronchocentric IAFE & $11 / 12$ \\
\hline Perilobular IAFE & $6 / 12$ \\
\hline Co-existent ILD in lower lobes & $7 / 7$ \\
\hline IAFE & $3 / 7$ \\
\hline IAFE and HP & $1 / 7$ \\
\hline UIP & $3 / 7$ \\
\hline \multicolumn{2}{|l|}{ Other features } \\
\hline Venous and arterial intimal fibrosis & $8 / 12$ \\
\hline Granulomas & $3 / 12$ \\
\hline Pleural ossification/calcification & $0 / 12$ \\
\hline
\end{tabular}

Data are presented as $\mathrm{n} / \mathrm{N}$ or range, unless otherwise stated. ILD: interstitial lung disease; PPFE: pleuroparenchymal fibroelastosis; NSIP: nonspecific interstitial pneumonia; UIP: usual interstitial pneumonia; $\mathrm{PAH}$ : pulmonary arterial hypertension; IAF: intra-alveolar fibrosis; IAFE: intra-alveolar fibrosis and elastosis; HP: hypersensitivity pneumonitis. ${ }^{\#}: 1$, minimal; 2 , moderate; 3 , severe. " : all zones. ${ }^{+}$: diffuse. ${ }^{\text {s: }}$ lower zone, two out of 12 ; middle/lower zone,

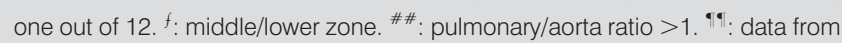
upper lobe biopsies in 11 out of 12 patients; one patient had sampling of middle and lower lobes only.

fibrosis with septal elastosis (IAFE) (12 out of 12 patients), with 11 out of 12 showing fibrosis of the pleura (fig. 5). Other upperlobe changes identified included foci of perilobular (six out of 12) and bronchocentric (10 out of 12) IAFE (fig. 6). Partial stenosis of pulmonary vasculature, both arterial and venous, was identified in eight out of 12 patients within the fibrotic areas; in two cases, this was sufficient to prompt suggestion of vasculo-occlusive disease in the original reports (fig. 7). Granulomatous inflammation was identified in three patients: in two it was localised, and in one there was a mixture of localised granulomas and bronchocentric palisading inflammation (fig. 7) thought to reflect superimposed ABPA, although no fungi were identified. All cases showed variable degrees of nonspecific chronic inflammation, focally with lymphoid follicle formation.

\begin{tabular}{|c|c|c|}
\hline TABLE 4 & \multicolumn{2}{|c|}{$\begin{array}{l}\text { Correlative findings of interstitial lung disease } \\
\text { (ILD) outside of upper lobes }\end{array}$} \\
\hline \multirow[t]{2}{*}{ Case } & \multicolumn{2}{|c|}{ Features of ILD outside upper lobes } \\
\hline & Imaging & Pathology $\#$ \\
\hline 1 & No & $\mathrm{Yes}^{\# \#}$ \\
\hline 2 & Yes & No second-site biopsy \\
\hline 3 & Yes $^{+}$ & Yes \\
\hline 4 & No & No second-site biopsy \\
\hline 5 & No & No second-site biopsy \\
\hline 6 & Yes $^{\S}$ & Yes \\
\hline 7 & Yes $^{\S}$ & $\mathrm{Yes}^{++}$ \\
\hline 8 & Yes $^{f}$ & No second-site biopsy \\
\hline 9 & No & Yes $^{\# \#}$ \\
\hline 10 & No & $Y e s^{\S \S}$ \\
\hline 11 & No & Yes $^{\# \#}$ \\
\hline 12 & Yes $^{\S}$ & No second-site biopsy \\
\hline \multicolumn{3}{|c|}{ 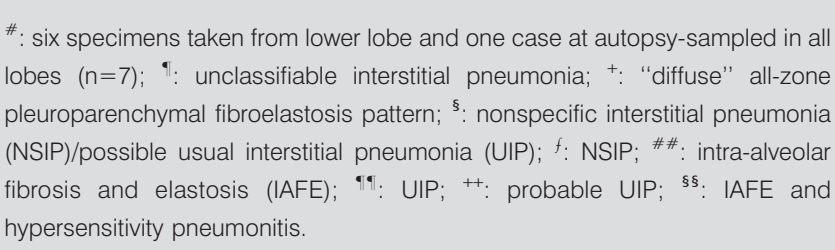 } \\
\hline
\end{tabular}

Six patients had second biopsies, all from the lower lobe, with the one case diagnosed at autopsy also being sampled from both lower lobes. All seven showed additional fibrosing lung disease. Four cases showed IAFE morphologically identical to, although less marked than, changes seen in the upper lobes in four cases, and three cases showed a pattern of UIP (fig. 8). In one of the patients with features of IAFE in the lower lobes, there were coexistent features of hypersensitivity pneumonitis comprising bronchocentric chronic inflammation and focal organising pneumonia, together with small, poorly formed non-necrotising granulomas. Of note, the IAFE appeared more bronchocentric in the lower-lobe biopsies with less pleural thickening.

\section{Imaging-pathology correlation}

Three out of six patients with HRCT features of co-existent fibrosis (NSIP/possible UIP radiological pattern, $n=2$; diffuse PPFE radiological pattern, $\mathrm{n}=1$ ) underwent biopsy and were histologically confirmed to have co-existent UIP. Three patients with features of lower-lobe fibrosis on HRCT did not have a lower-lobe biopsy, with HRCT patterns resembling NSIP $(n=1)$, NSIP/possible UIP $(n=1)$ and unclassifiable interstitial pneumonia $(\mathrm{n}=1)$.

Four patients who demonstrated features of distant ILD histologically had no corresponding HRCT abnormality. The histological pattern for three of these four patients was that of PPFE, represented by IAFE on histology (cases 1, 9 and 11). The fourth patient had histological features suggesting both diffuse PPFE and hypersensitivity pneumonitis (case 10).

\section{Treatment}

Complete treatment records were available in nine out of 12 patients. All were treated with courses of low-dose corticosteroids 

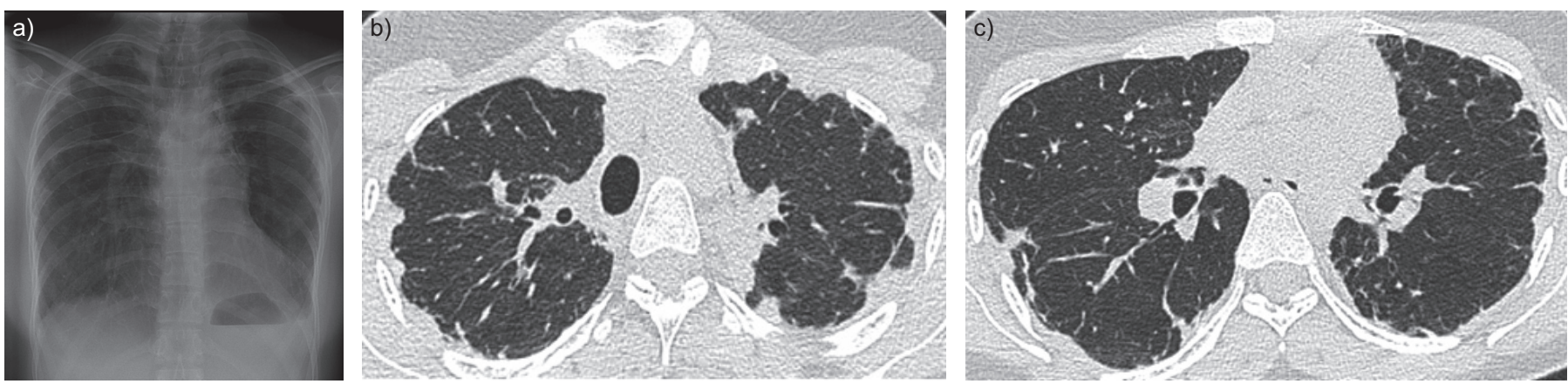

FIGURE 1. Case 4,35-yr-old female. a) Chest radiograph showing bilateral apical irregular pleural thickening. There is a pneumomediastinum and upper lobe volume loss with hilar elevation. High-resolution computed tomography through the b) upper and c) middle zones showing typical features of pleuroparenchymal fibroelastosis, with bilateral irregular pleural thickening and a subjacent reticular pattern consistent with fibrosis.

in the form of oral prednisone, with two of these patients receiving additional high-dose corticosteroids in the form of pulsed intravenous methylprednisone. Two patients received additional immunosuppressant therapy in the form of cyclophosphamide $(n=1)$ and azathioprine $(n=1)$. Two patients were treated with $\mathrm{N}$-acetylcysteine. One patient received both immunosuppressant therapy (cyclophosphamide) and $N$-acetylcysteine. Two patients received prophylactic antibiotics (azithromycin) for
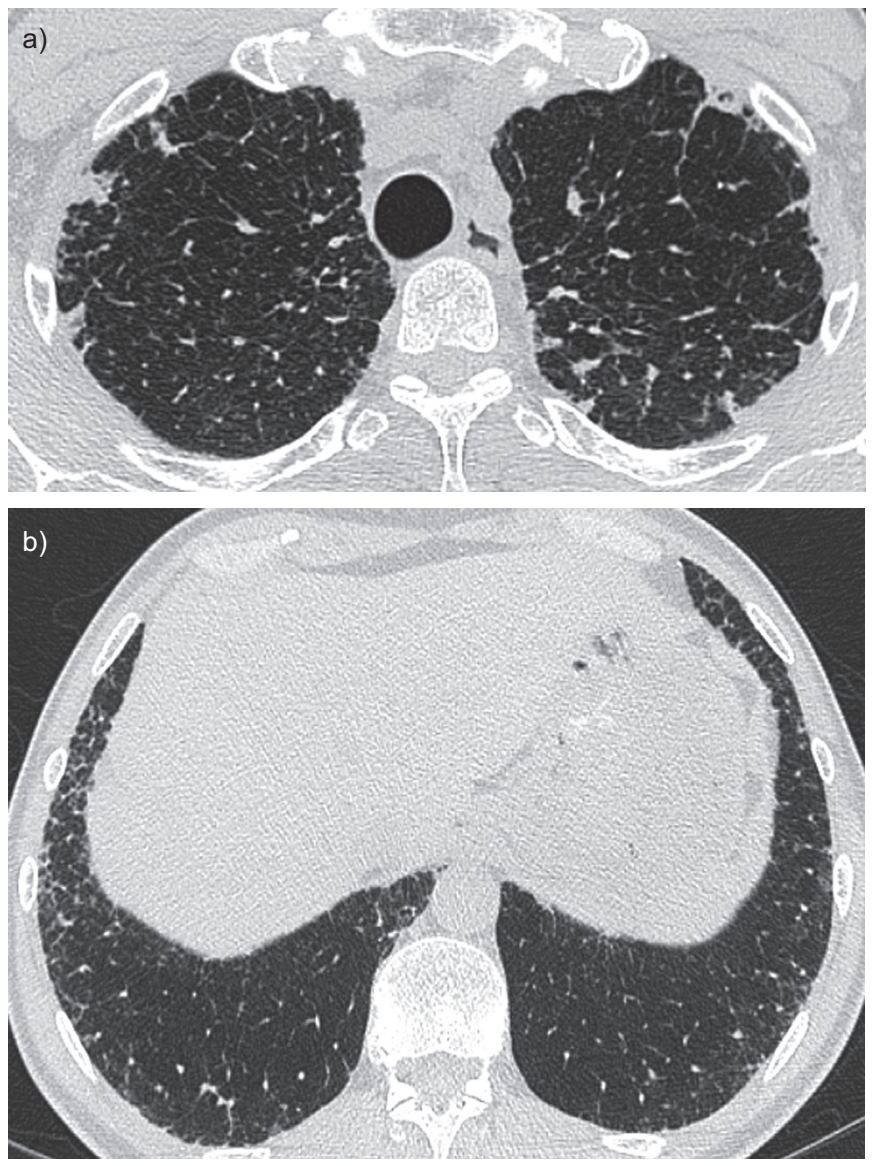

FIGURE 2. Case 6, 59-yr-old male. a) High-resolution computed tomography through the upper lobes, demonstrating features of pleuroparenchymal fibroelastosis. b) Subpleural reticulation in the lower lobes, consistent with nonspecific interstitial pneumonia/atypical usual interstitial pneumonia. recurrent infections. One patient, whose biopsy showed suspected superimposed ABPA, was treated with antifungal agents (case 10) and showed response to therapy.

Of the 10 patients with follow-up data, seven demonstrated disease progression. Five of these patients died, with the time interval between diagnosis of PPFE and death ranging from 4 months to 2 yrs. Four of these five patients reported recurrent infections during the course of their disease. One of the
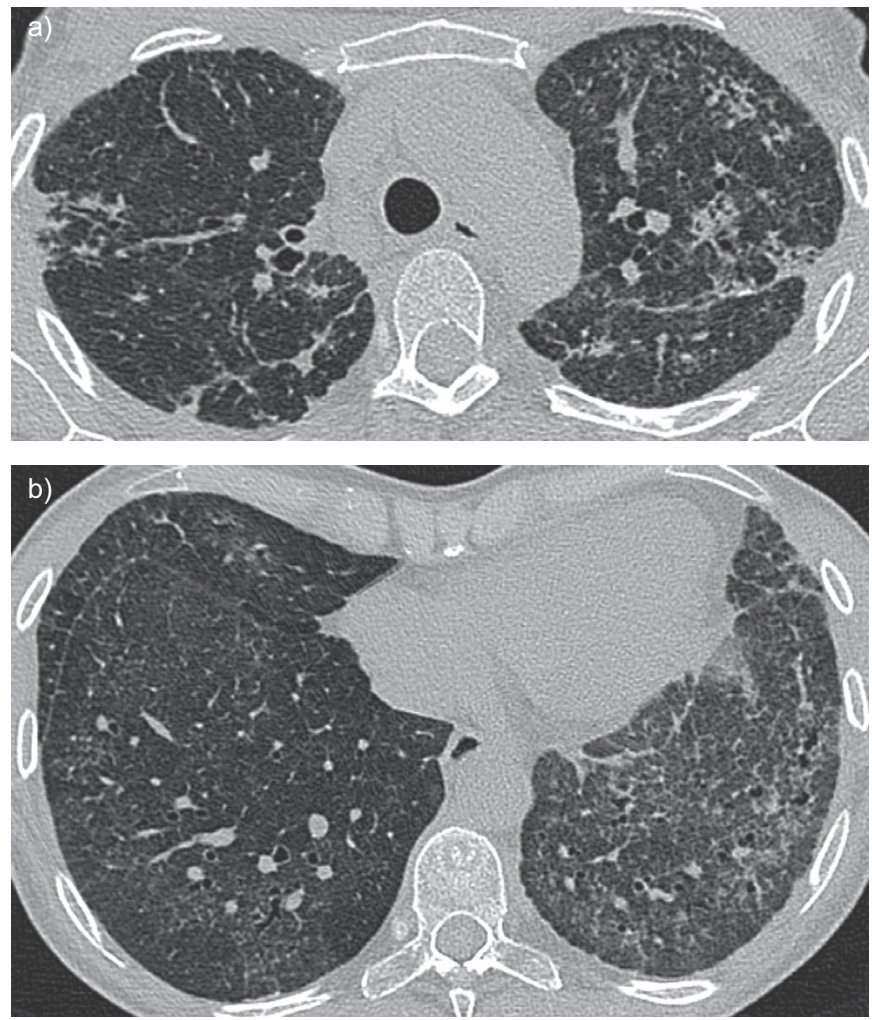

FIGURE 3. Case 8, 57-yr-old female. a) High-resolution computed tomography (HRCT) through the upper lobes. In addition to features consistent with pleuroparenchymal fibroelastosis, there is a reticular pattern, patchy ground-glass change and associated traction bronchiectasis in the subjacent parenchyma. b) HRCT through the lower lobes showing widespread fine reticulation and groundglass opacification, reminiscent of fibrotic nonspecific interstitial pneumonia. 

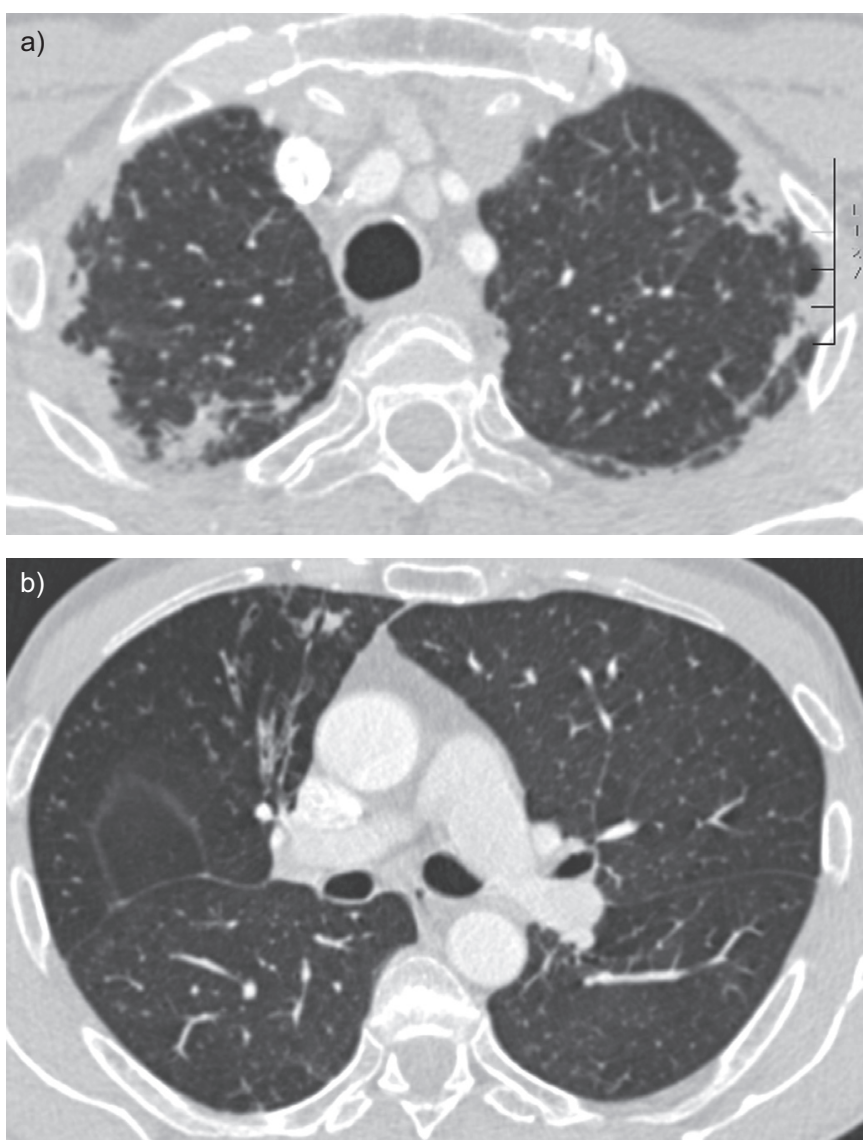

FIGURE 4. Case 9, 65-yr-old male. a) Computed tomography section from a contrast-enhanced study showing features of pleuroparenchymal fibroelastosis at the lung apices. b) Anterior-segment, right upper lobe cylindrical bronchiectasis with mucous plugging; on other sections, there was a background of mosaic attenuation reflecting small-airway obliteration.

five patients tested positive for Aspergillus sp. Four of these patients also had positive autoantibody screens.

Both patients whose clinical courses were stable demonstrated features of co-existent fibrosis. The patient with HRCT features resembling NSIP/possible UIP underwent biopsy, which was histologically confirmed as UIP, while the patient with HRCT features of nonspecific fibrosis did not undergo a lower-lobe biopsy.
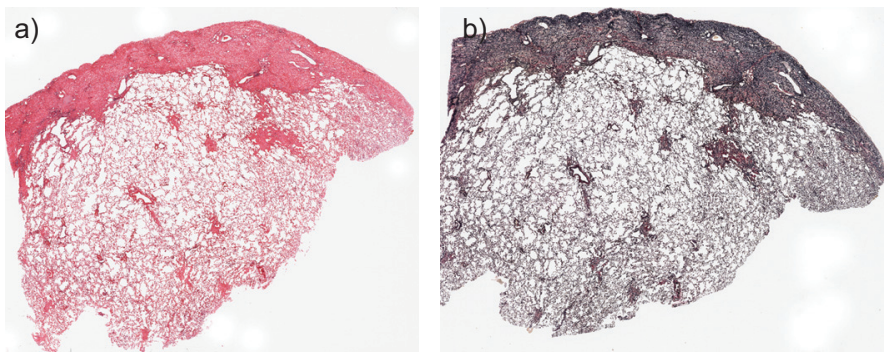

\section{DISCUSSION}

This study describes a group of patients who fulfilled published histopathological and imaging criteria for PPFE. Review of our clinical data confirms that this condition appears to be a distinct clinicopathological entity, although $25 \%$ had coexistent UIP in the lower lobes and other cases showed features of PPFE in other zones, either on HRCT or lung biopsy.

Although only rarely reported in the English literature [2, 3], PPFE is more frequently described by Japanese groups [1, 4-5, 10-14]; a meta-analysis of the available literature (table 5) shows a male/female ratio of $2 / 1$, compared with our series where there was a male/female ratio of $1 / 1.4$. The median age of patients from previous studies was $46 \mathrm{yrs}$, which is younger than in our series, in which the median age was 57 yrs. Comparable results were obtained with respect to smoking history, with both previous and current series reporting "never" smoking histories in the majority $(62 \%$ and $75 \%$, respectively).

In terms of aetiology, there are several observations that may be of relevance to cause and/or association, in what is usually considered to be an idiopathic condition. First, just over half of patients reported recurrent infections during the course of their disease. This is higher than figures reported by AMITANI et al. [1] (23\%) and FRANKEL et al. [2] (40\%). One of these patients had superimposed ABPA, evidenced by histology, serology and response to therapy. Furthermore, one case rejected on pathology review but with definite computed tomography features had background ABPA in association with a co-existent aspergilloma, for which a resection had been undertaken (the latter being the reason for exclusion). PICIUCCHI et al. [15] recently reported a case of a patient who tested positive for Aspergillus precipitins. Viewing these data collectively, it can be speculated that repeated inflammatory damage in a predisposed individual may lead to this pattern of IAFE. Localised pleural thickening has been reported in patients with ABPA and cystic fibrosis, lending support to this theory [16-19].

Secondly, five patients demonstrated autoantibodies suggesting that autoimmunity may be a factor in some patients. Another patient had previously been diagnosed with Dressler's syndrome, a condition with an autoimmune basis [20-22]. One patient who demonstrated high levels of autoantibodies had a history of renal transplant. Features of PPFE have been described in patients after bone marrow transplantation [7],

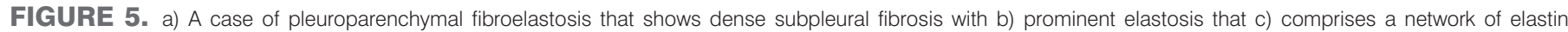

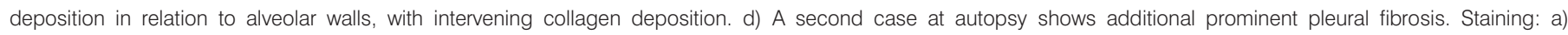
haematoxylin and eosin; b-d) elastic Van Gieson staining. Magnification: a, b, d) $2 \times$; c) $100 \times$ 

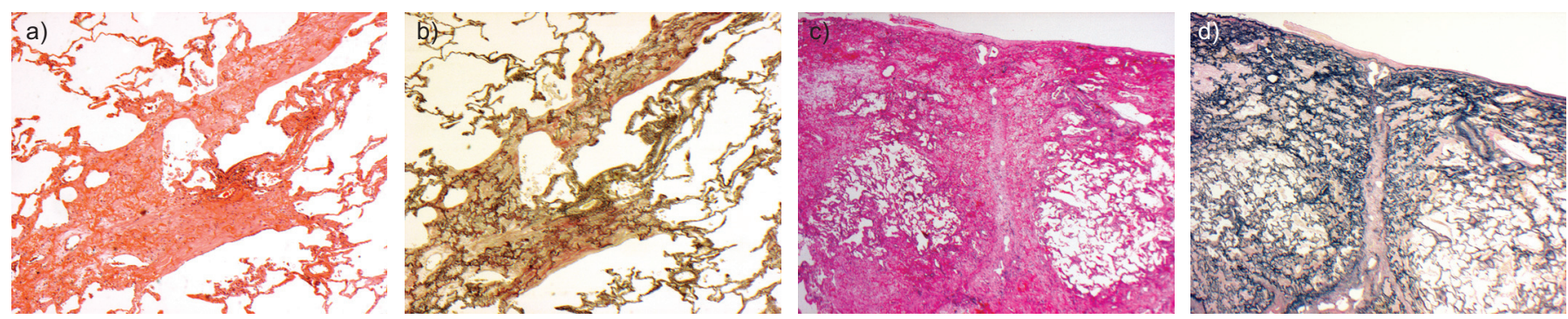

FIGURE 6. a, b) Intra-alveolar fibrosis distant from the pleura shows a peribronchiolar distribution, extending to surround an alveolar duct (top right). c, d) Other areas shows a more perilobular distribution as the intra-alveolar fibrosis extends away from the pleura. Staining: a, c) haematoxylin and eosin; b, d) elastic Van Gieson. Magnification: a, b) $100 \times c$, d) $20 \times$

further supporting an autoimmune component to the development of disease.

Genetic predisposition is probably a further factor. AzOULAY et al. [6] reported bilateral apical pleural fibrosis in three siblings, in whom an underlying aetiology was not determined. In our series, two out of 12 patients had a family history of ILD, a lower frequency than other published series (AMATANi et al. [1], 30\%; FRANKel et al. [2], 40\%; SHIOTA et al. [4], 57\%).

None of these data points to a single cause and the majority of cases of PPFE will probably continue to be viewed as idiopathic. However, our findings suggest that cases diagnosed with PPFE should be investigated for features of infection (in particular aspergillosis), autoimmunity and a family history, all of which may influence management.

Our series of patients with PPFE had a high prevalence of coexistent ILD. Of four patients with a different histological pattern, two (biopsy-confirmed UIP) had features of coexistent interstitial fibrosis on computed tomography. Four other patients who demonstrated imaging features of lowerlobe fibrosis did not undergo biopsy of a second site. It is interesting to note that the lower-lobe (UIP) fibrosis progressed in two cases with serial imaging while the areas of PPFE in the upper lobes remained stable. In addition, four out of the five patients with nonspecific autoantibody positivity demonstrated imaging features of distant fibrosis during the course of their disease. It is estimated that up to $15-20 \%$ of patients who present with a chronic ILD either have an occult connective tissue disease or subsequently develop a clinically overt connective tissue disease [23] and histological features similar to those of PPFE are reported in patients with apical fibrosis known to have ankylosing spondylitis [24, 25]. Increased levels of autoantibodies have also been reported in studies of patients with interstitial pulmonary fibrosis (IPF), although it has been suggested that the presence of autoantibodies in IPF patients may represent a nonspecific result secondary to lung inflammation and injury [26, 27]. Finally, although not described in the series from North America [2], co-existent fibrosis, including UIP, has occasionally been described in the more extensive Japanese literature $[4-5,10,12]$. Taken together, these data support the concept that patients may have a genetic predisposition to lung fibrosis, which may manifest as different histopathological patterns of fibrosing lung disease.

In terms of histopathology, but not computed tomography, features of PPFE were also found in the lower lobes of four out of seven patients, although there was a greater extent of bronchocentricity and the disease was milder in nature. It can be therefore be argued that this lends further support to the idea that airway-centred damage may be key to the pathogenesis of this disorder, as discussed above in relation to the aetiology of PPFE.

Our observations highlight some key concepts regarding integration of pathology into the multidisciplinary team (MDT)
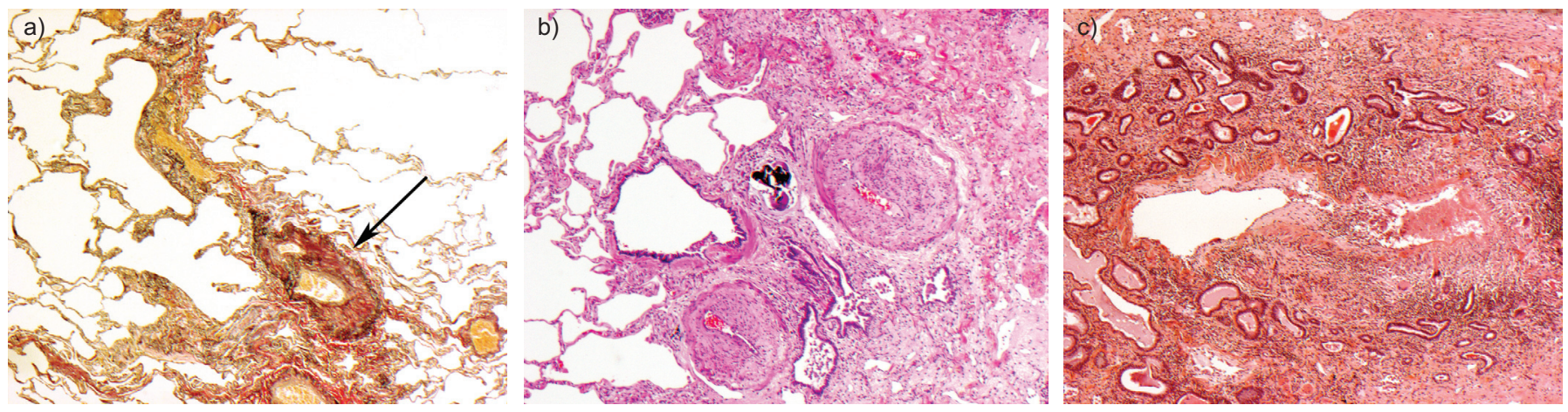

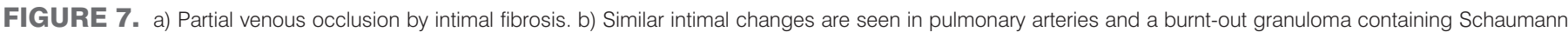

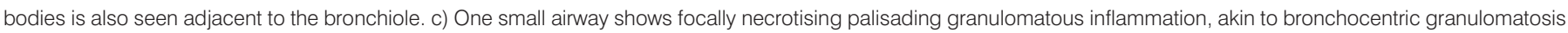
Staining: a) elastic Van Gieson; b, c) haematoxylin and eosin. Magnification: a, b) $100 \times$; c) $40 \times$. 

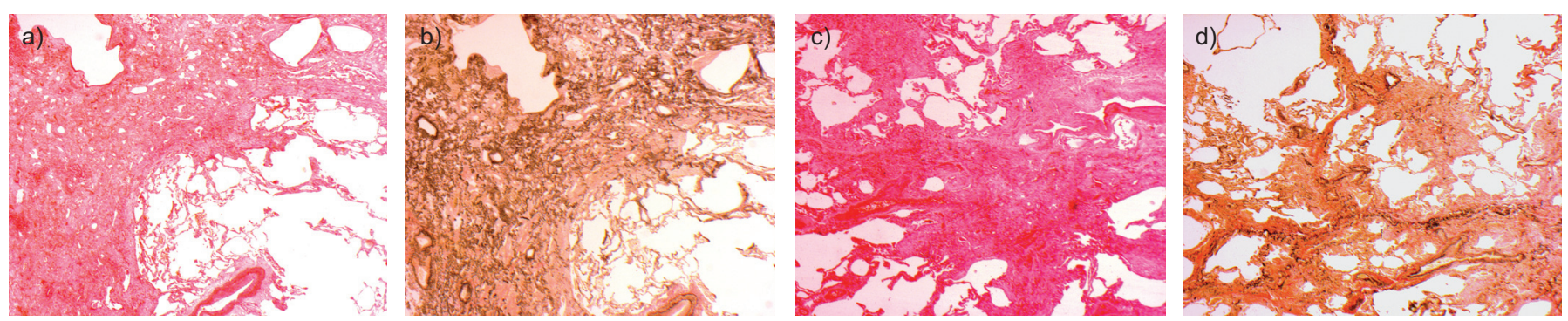

FIGURE 8. A case of combined pleuroparenchymal fibroelastosis and usual interstitial pneumonia (UIP). a, b) A biopsy from the upper lobe shows patchy established fibrosis with areas of fibroblastic change at its edge. Elastic Von Giesen (EVG) staining shows that the fibrosis is intra-alveolar, with elastosis marking the residuum of the alveolar walls. c, d) The biopsy from the lower lobe of the same patient shows a patchier fibrosis, again with fibroblastic change, but the EVG staining shows sparser, more fragmented and more disorganised elastin staining, typical of UIP. Staining: a, c) haematoxylin and eosin; b, d) EVG. Magnification: $40 \times$

management of patients with diffuse lung disease and the importance of ensuring that multiple biopsies are taken from different areas of concern. One patient did not have a biopsy taken from the upper lobes, where disease was most marked on computed tomography, and the diagnosis was only made confidently after MDT review. In other cases with co-existent fibrosis identified on computed tomography, biopsies were not taken from the lower lobes; thus, it remains uncertain whether these areas were an extension of PPFE to the lower lobes or represent another disease, such as UIP/IPF, with attendant differences in treatment and prognosis.

It should be emphasised that IAFE is not specific to PPFE and can be seen as a result of radiotherapy, following chemotherapy and as a sequel to certain inhalational injuries. The pathogenesis of IAFE remains poorly understood but clearly it is a pathway of lung injury common to a variety of disorders, including PPFE. The features of IAFE also overlap with those of an apical cap [28, 29]. However, apical caps are anatomically localised, with no extension to other lobes so, apart from rare cases that form mass lesions, these should not enter the differential diagnosis of PPFE since a parenchymal component is not an imaging feature of an apical cap.

Treatment administered to patients in this series was highly variable and largely empirical, reflecting the lack of experience in treating patients with PPFE. The clinical course was progressive in many of these patients, a finding concordant with previous studies, despite aggressive treatment in some cases, including corticosteroids and immunosuppressants.

Limitations of this case series are its retrospective nature and the fact the data are not entirely complete, but we believe that this cohort, the largest in Western literature to date, confirms PPFE as a distinct clinicopathological entity. In view of our stringent inclusion criteria, the number in our "pure" cohort was smaller than it might otherwise have been. Arguably, one of our cases could, in retrospect, have been excluded given the potential overlap with ABPA, but we chose to keep it within the series to emphasise the point regarding potential clinical associations.

TABLE 5 Meta-analysis of the literature: clinical data

\begin{tabular}{|c|c|c|c|c|c|c|c|c|c|c|}
\hline First author [ref.] & Year & $\begin{array}{c}\text { Age range } \\
\text { yrs }\end{array}$ & Sex & $\begin{array}{l}\text { Recurrent } \\
\text { infection }\end{array}$ & PT/PM & Smoking $\mathrm{Hx}^{\top}$ & Occ/Env Hx & Fam Hx & $\begin{array}{l}\text { Clinical } \\
\text { course }^{+}\end{array}$ & Deaths $n$ \\
\hline SнIота [4] & 1999 & $25-83$ & $5 / 2$ & 1 & 3 & 2/2/3 & 1 & 4 & $0 / 1 / 6$ & 3 \\
\hline КоBAYASHI [5] & 1999 & 27 & $1 / 0$ & 0 & 1 & 0/0/1 & 0 & 1 & $0 / 1 / 0$ & 0 \\
\hline JUNGU [11] & 1999 & $39-43$ & $0 / 2$ & 0 & 2 & 0/0/2 & 0 & 0 & $0 / 1 / 1$ & 0 \\
\hline NEI [13] & 2006 & 82 & $1 / 0$ & 0 & 0 & $0 / 1 / 0$ & 0 & 0 & 0/0/1 & 1 \\
\hline Мовімото [14] & 2010 & 49 & $1 / 0$ & 0 & 0 & $0 / 1 / 0$ & 0 & 0 & Unknown & 0 \\
\hline AZOULAY [6] & 1999 & 23-29 & $0 / 3$ & 0 & 3 & 0/0/3 & 0 & 3 & 0/0/3 & 2 \\
\hline FRANKEL [2] & 2004 & $32-65$ & $2 / 3$ & 2 & 0 & $0 / 1 / 4$ & 0 & 2 & 0/0/2 & 2 \\
\hline BECKER [3] & 2008 & $51-59$ & $0 / 2$ & 0 & 1 & $0 / 2 / 0$ & 1 & 0 & $0 / 0 / 1$ & 1 \\
\hline РıсIUсCHI [15] & 2011 & $28-68$ & $2 / 0$ & 0 & Unknown & $0 / 0 / 2$ & 1 & 0 & Unknown & 0 \\
\hline
\end{tabular}

PT: pneumothorax; PM: pneumomediastinum; Hx: history; Occ/Env: occupational/environmental allergen exposure; Fam: family. *: data are presented as numbers of males/females; " : data are presented as numbers of current/ex-/never-smokers; ${ }^{+}$: data are presented as numbers of patients whose condition improved/remained stable/ deteriorated; ${ }^{\S}$ : median 46 yrs. 
In conclusion, we have described twelve patients with PPFE, the disease being more widespread in terms of zonal involvement than previously reported. Indirect data suggest that recurrent infections may have a role in pathogenesis, perhaps with contributions from genetic predisposition and autoimmune mechanisms. The fact that a number of patients with PPFE demonstrated features of coexistent interstitial lung disease should alert the referring clinician to consider both diffuse forms of PPFE and other fibrosing lung diseases in the differential diagnosis.

\section{STATEMENT OF INTEREST}

None declared.

\section{REFERENCES}

1 Amitani R, Niimi A, Kuse F. [Idiopathic pulmonary upper lobe fibrosis (IPUF)]. Kokyu 1992; 11: 693-699.

2 Frankel SK, Cool CD, Lynch DA, et al. Idiopathic pleuroparenchymal fibroelastosis: description of a novel clinicopathologic entity. Chest 2004; 126: 2007-2013.

3 Becker CD, Gil J, Padilla ML. Idiopathic pleuroparenchymal fibroelastosis: an unrecognized or misdiagnosed entity? Mod Pathol 2008; 21: 784-787.

4 Shiota S, Shimizu K, Suzuki M, et al. [Seven cases of marked pulmonary fibrosis in the upper lobe]. Nihon Kokyuki Gakkai Zasshi 1999; 37: 87-96.

5 Kobayashi Y, Sakurai M, Kushiya M, et al. [Idiopathic pulmonary fibrosis of the upper lobe: a case report]. Nihon Kokyuki Gakkai Zasshi 1999; 37: 812-816.

6 Azoulay E, Paugam B, Heymann MF, et al. Familial extensive idiopathic bilateral pleural fibrosis. Eur Respir J 1999; 14: 971-973.

7 von der Thusen JH, Hansell DM, Veys PA, et al. Pleuroparenchymal fibroelastosis in patients with pulmonary disease secondary to bone marrow transplantation. Mod Pathol 2011; 24: 1633-1639.

8 American Thoracic Society, European Respiratory Society. American Thoracic Society/European Respiratory Society International Multidisciplinary Consensus Classification of the Idiopathic Interstitial Pneumonias. Am J Respir Crit Care Med 2002; 165: 277-304.

9 Raghu G, Collard H, Egan J, et al. An official ATS/ERS/JRS/ALAT statement: idiopathic pulmonary fibrosis: evidence-based guidelines for diagnosis and management. Am J Respir Crit Care Med 2011; 183: 788-824.

10 Sakamoto A, Kuwano K, Komori M, et al. [A case with usual interstitial pneumonia characterised by subpleural fibrosis predominantly in the bilateral upper lobes]. Nihon Kyobu Rinsho 2000; 59: 628-631.

11 Jungu K, Kawana A, Furihata K, et al. Two cases of marked pulmonary fibrosis in the upper lung field. Kokyu 1999; 18: 318-323.
12 Kobashi Y, Ohbai H, Yoneyama, et al. [A case of so-called "idiopathic pulmonary upper lobe fibrosis" complicated by both mediastinal emphysema and bilateral pneumothorax at different times]. Kokyu 2000; 19: 292-298.

13 Nei T, Kawamoto M, Satoh E, et al. [A case of suspected idiopathic pulmonary upper lobe fibrosis (Amitani disease) with acute exacerbation]. Nihon Kokyuki Gakkai Zasshi 2009; 47: 116-121.

14 Morimoto A, Mochizuki Y, Nakahara Y, et al. [Case of idiopathic pulmonary upper lobe fibrosis]. Nihon Kokyuki Gakkai Zasshi 2010; 48: 944-949.

15 Piciucchi S, Tomassetti D, Casoni G, et al. High resolution CT and histological findings in idiopathic pleuroparenchymal fibroelastosis: features and differential diagnosis. Respir Res 2011; 12: $1-5$.

16 Denning DW, Riniotis K, Dobrashian R, et al. Chronic cavitary and fibrosing pulmonary and pleural aspergillosus: a case series, proposed nomenclature change, and review. Clin Infect Dis 2003; 37: Suppl. 3, S265-S280.

17 Franquet T, Gimenez A, Cremades R, et al. Spontaneous reversibility of "pleural thickening" in a patient with semi-invasive pulmonary aspergillosis: radiographic and CT findings. Eur Radiol 2000; 10: 722-724.

18 Hansell DM, Strickland B. High-resolution computed tomography in pulmonary cystic fibrosis. Br J Radiol 1989; 62: 1-5.

19 Angus RM, Davies ML, Cowan MD, et al. Computed tomographic scanning of the lung in patients with allergic bronchopulmonary aspergillosis and in asthmatic patients with a positive skin test to Aspergillus fumigatus. Thorax 1994; 49: 586-589.

20 Bendjelid K, Pugin J. Is Dressler syndrome dead? Chest 2004; 126 : 1680-1682.

21 Earis JE, Marcuson EC, Bernstein A. Complement activation after myocardial infarction. Chest 1985; 87: 186-190.

22 Wessman DE, Stafford CM. The postcardiac injury syndrome: a case report and review of the literature. South Med J 2006; 99: 309-314.

23 Tzelepis GE, Toya SP, Moutsopoulos HM. Occult connective tissue diseases mimicking idiopathic interstitial pneumonias. Eur Respir J 2008; 31: 11-20.

24 Jessamine AG. Upper lung fibrosis in ankylosing spondylitis. CMAJ 1968; 98: 25-29.

25 El Maghraoui A. Pleuropulmonary involvement in ankylosing spondylitis. Joint Bone Spine 2005; 72: 496-502.

26 Singh S, du Bois R. Autoantibodies in cryptogenic fibrosing alveolitis. Respir Res 2001; 2: 61-63.

27 Schattner A, Aviel-Ronen S, Mark EJ. Accelerated usual interstitial pneumonitis, anti-DNA antibodies and hypocomplementemia. J Intern Med 2003; 254: 193-196.

28 Yousem SA. Pulmonary apical cap: a distinctive but poorly recognised lesion in pulmonary surgical pathology. Am J Surg Pathol 2001; 25: 679-683.

29 Butler C, Kleinerman J. The pulmonary apical cap. Am J Pathol 1970; 60: 205-216. 